\title{
HST Study of the Molecular Gas in Planetary Nebulae
}

\author{
Josh Tartar ${ }^{1}$, Sarah Eyermann ${ }^{1}$, Angela Speck ${ }^{1}$ \\ and Margaret Meixner ${ }^{2}$ \\ ${ }^{1}$ Department of Physics and Astronomy, University of Missouri-Columbia, Columbia, \\ MO 65201, USA \\ email: tartarj@missouri.edu \\ ${ }^{2}$ Space Telescope Science Institute, 3700 San Martin Drive, Baltimore, MD 21218
}

\begin{abstract}
A better understanding of the nature of the molecular and ionized gas envelopes of planetary nebulae $(\mathrm{PNe})$ is important both to our understanding of their contribution to the ISM and to the evolution of PNe themselves. Using HST's unprecedented resolution and sensitivity for near-IR observations, we are studying the distribution and intensity of molecular gas emission from PNe.
\end{abstract}

Keywords. ISM: planetary nebulae: general, ISM: planetary nebulae: individual (He 3-1475, Hb 12, BD+30 3639, NGC 7027, NGC 6720, NGC 7293), stars: AGB and post-AGB

\section{Introduction}

The six PNe we are studying, in order of dynamical age are: Hen 3-1475, Hb 12, NGC 7027, BD+303639, NGC 6720, and NGC 7293. In all cases, $\mathrm{H}_{2}$ is present within the ionized nebula. The $\mathrm{H}_{2}$ appears in a thin shell around the central star. However, $\mathrm{Hb} 12$ is the nebula whose $\mathrm{H}_{2}$ emission morphology is markedly different from its $\mathrm{H} \alpha$ morphology; Figs. 1 and 2. The $\mathrm{H} \alpha$ image of $\mathrm{Hb} 12$ shows an hourglass-shaped bipolar nebula. The waist of this hourglass shape is assumed to be the dense dust torus that is expected to form towards the end of the AGB. In the $\mathrm{H}_{2}$ image of $\mathrm{Hb} 12$, the center is extremely bright, and is surrouded by an "eye-shaped" band of emission, which we interpret as a ring of molecular gas at a non-zero inclination angle. This outer ring of molecular gas does not quite lie in the same plane as the dense inner torus. This unresolved inner $\mathrm{H}_{2}$ emission demonstrates that mass loss hass been episodic.

Table 1.

\begin{tabular}{lcccccc}
\hline PN & $\begin{array}{c}\text { Age } \\
\left(10^{3} \mathrm{yrs}\right)\end{array}$ & $\begin{array}{c}\text { Distance Shortest radius } \\
(\mathrm{kpc})\end{array}$ & $\begin{array}{c}\text { Longest radius } \\
\left(\times 10^{17} \mathrm{~cm}\right)\end{array}$ & $\begin{array}{c}\text { Viewing } v_{\exp } \\
\text { Angle }\end{array}\left(\mathrm{km} \mathrm{s}^{-1}\right)$ \\
\hline Hen 3-1475 & & $2.5^{d}$ & $\mathrm{n} / \mathrm{a}$ & $\mathrm{n} / \mathrm{a}$ & $\mathrm{n} / \mathrm{a}$ & \\
Hb12 & $0.3^{a}$ & $3^{e}$ & 1.2 & 2.4 & $60^{\circ}$ & \\
NGC 7027 & $0.9^{b}$ & $0.7^{e}$ & 0.47 & 0.68 & $46.3^{\circ}$ & \\
BD+303639 & $0.9^{c}$ & $1.5^{e}$ & 0.45 & 0.72 & $51.3^{\circ}$ & \\
NGC 6720 & $1.6^{c}$ & $0.7^{c}$ & 2.5 & 3.4 & $42.7^{\circ}$ & $45^{c}$ \\
NGC 7293 & $10.6^{c}$ & $0.2^{c}$ & 9.2 & 11.4 & $36.2^{\circ}$ & $31^{c}$ \\
\hline
\end{tabular}

${ }^{b}$ Latter et al. (2000); ${ }^{c}$ O'Dell et al. (2002); ${ }^{d}$ Bobrowsky (1993); ${ }^{e}$ Liu et al. (2001). 


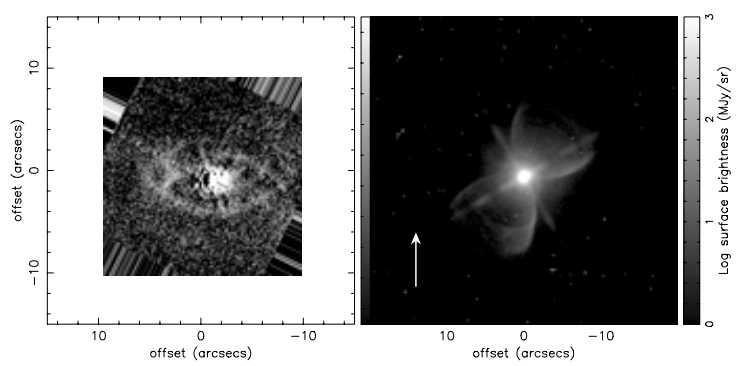

Figure 1. Figures 1a (left): NICMOS2 $\mathrm{H}_{2}$ image of Hubble 12 at $2.12 \mu \mathrm{m}$ and $1 \mathrm{~b}$ (right): WFPC2 $\mathrm{H} \alpha$ image of Hubble 12. Up arrow indicates north for both images.

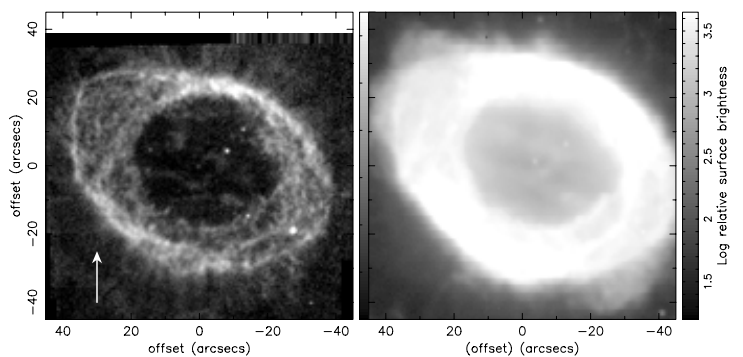

Figure 2. Figures 2a (left): NICMOS2 $\mathrm{H}_{2}$ image of BD+30 3639 at $2.12 \mu \mathrm{m}$ and $1 \mathrm{~b}$ (right):

WFPC2 $\mathrm{H} \alpha$ image of $\mathrm{BD}+303639$. Up arrow indicates north for both images.

\section{Morphologies}

We assume that each PN is surrounded by a circular ring of molecular gas, and that the observed shapes around each object are due to the viewing angle. We have determined the extent and inclination angles for the rings of molecular emission in each nebula. Assuming a constant outflow velocity the dimensions of the molecular rings translate into timescales, as shown in Table 1 . This assumes that the long radius is the actual radius of the ring, and the short radius is an effect of the viewing angle. Here, 0 degrees is defined as the angle that would make the $\mathrm{H}_{2}$ ring parallel with the plane of the sky. Along with the extent of the ring of material, clumpiness of the molecular gas seems to change with age.

\section{References}

Bobrowsky, 1993, BAAS, 25, 783128, 2339.

Latter, W.B. et al. 2000, ApJ, 539, 783.

Lui, W.B. et al. 2001, MNRAS, 323, 343.

O'Dell, C.R. et al. 2002, AJ, 123, 3329. 